\title{
Axial and para-axial loading response evaluation on human cadaver-harvested lumbar vertebral blocks: In vitro experiment with possible clinical implications for clinical practice
}

\author{
DAN MARCEL ILIESCU ${ }^{1 *}$, SERGIU-IOAN MICU ${ }^{2}$, COSTIN IONESCU $^{1}$, IONUT BULBUC ${ }^{1}$, PETRU BORDEI $^{1}$, \\ BOGDAN OBADA $^{1,3}$, FELIX VOINEA ${ }^{4}$, EMMA GHEORGHE $^{1^{*}}$ and MADALINA GABRIELA ILIESCU ${ }^{1,5}$ \\ ${ }^{1}$ Department of Anatomy and Histology, Faculty of Medicine, 'Ovidius' University of Constanta, \\ 900527 Constanta; Departments of ${ }^{2}$ Gastroenterology, ${ }^{3}$ Orthopaedic Surgery and ${ }^{4}$ Urology, \\ Clinical County Hospital of Constanta, 900591 Constanta; ${ }^{5}$ Department of Balneo-Physiotherapy \\ and Recuperation, Balneal Recovery Sanatorium of Techirghiol, 906100 Constanta, Romania
}

Received June 15, 2021; Accepted July 15, 2021

DOI: $10.3892 /$ etm.2021.10626

\begin{abstract}
The aim of the present study conducted on the lumbar spine was to confirm that the pronounced decrease in resistance in the system is a phenomenon that can be eminently affected by the adaptive changes that occur at the level of the intervertebral disc at axial mechanical stresses. The biomechanical trial was carried out on 11 lumbar segments L1-L5, gathered from adult human cadavers. The dissection considered the complete keeping of all bone, disc, articulated and ligamentous components in their anatomical position. All 11 samples were frozen $24 \mathrm{~h}$ prior to the performance of the biomechanical measurement. The specimens were placed in the testing device, their placement being conditioned by the estimated dimensional values. Thus, to calculate the load and axial resistance, the models were placed vertically, central between the test machine ferries. The testing was carried out by applying variable forces and displacement supervision. The displacement interval was represented by a segment of $0-10 \mathrm{~mm}$ with surveillance every $2 \mathrm{~mm}$. Mobility in the sagittal plane (flexion earlier in our case) was much higher than that in the frontal plane, obviously limiting mobility via the intervertebral disc and articular complex through the presence of arches. Statistical analysis demonstrated the lack of any correlation values between the two types of movements $\left(\mathrm{R}^{2}=0.005507\right)$, underlining the absence of any prediction elements. A noteworthy aspect is that the correlations appeared low, statistically insignificant, even
\end{abstract}

Correspondence to: Dr Sergiu-Ioan Micu, Department of Gastroenterology, Clinical County Hospital of Constanta, 145 Tomis Boulevard, 900591 Constanta, Romania

E-mail: micuioansergiu@yahoo.com

${ }^{*}$ Contributed equally

Key words: lumbar spine, mechanical stresses, vertebral blocks, axial load, eccentric load within the same movement in the sagittal plane between the two levels, L1-L3 and L3-L5 $\left(\mathrm{R}^{2}=0.610427\right)$, which may lead to the possibility of the emergence of significant differences in mobility between respective levels. The behavior type of the monitored specimens and the results obtained allowed the mapping of objective parallelism between the values obtained and the behavior in vivo of the lumbar vertebral segment.

\section{Introduction}

Experimental dynamics conducted on the lumbar spine aims to prove that, given that the vertebral bodies are rigid systems, the pronounced decrease in resistances in the system is a phenomenon that can be eminently affected by the adaptive changes that occur at the level of the intervertebral disc at axial mechanical stresses. Moreover, these considerations are applicable to the disc where degenerative processes have occurred $(1,2)$. However, it does not perfect the ways of distributing loads and demands that occur in the vertebral body and the intervertebral disc. Such a model, associated with the biodynamic axial load study, has the advantage of providing relevant information on bone movements and the changes in soft neighborhood structures. This approach should not be neglected in specific pathology management of the area (1). The behavior of the monitored specimens, especially the results, allows for objective parallelism between the values obtained in the present study and the lumbar vertebral segments in vivo behavior.

\section{Materials and methods}

The biomechanical trial included 11 lumbar segments L1-L5, gathered from adult human cadavers. To obtain various anatomical preparations with biomechanical characteristics as close to those, in vivo, specimens were obtained from fresh, non-formalin-preserved human cadavers, preserved by freezing at $-22^{\circ}$; after sampling, the anatomical preparations were maintained at temperatures below freezing. The decision to protect the samples only by freezing was taken after 
examining the specialists' studies from the literature and which confirmed, with arguments, that this represented the most effective preservation method concerning the mechanical strength and elasticity of the intervertebral disc for maintenance in saline solution, which decreases disc resistance (3). After the isolation, the adjacent muscularity was removed from the preparations to view the osteoligamentous spine fully. The dissection considered the complete keeping of all bone, disc, articulated and ligamentous elements in their anatomical position to maintain the mechanical characteristics specific to the region as close as possible to the physiological conditions. Preparations were frozen $24 \mathrm{~h}$ prior to the performance of the biomechanical measurement (3).

The test device included the following. i) Tensile strength-compression universal test machine, with the capacity of $200 \mathrm{kN}$, type LBG 200, with a rigid construction in 2 columns, for bending, compression, and tensile strength static tests, with an accuracy that observes the standards of ISO 7500-1, precision class 0.5 and minimal resolution 0.80 microns. The device has transmission channels of high resolution and synchronized data, with high precision power cells, with a piston displacement transducer with double direction. ii) Command system and digital control with software module through which the machine operation is performed, setting of work parameters occurs, and reading the results specific to the bending/compressing/tensile tests is carried out. The system ensures the real-time follow-up of test parameters: force, displacement, elongation and allows the printing of results and test diagrams, achieved using a digital regulator for force, displacement, deformation with closed-loop control, and data acquisition. iii) The programmable package on Windows platform; a programmable package for statistical tests or according to the operator needs, represented by TC SOFT application software (Microsoft Corp.).

Each vertebral segment under test was comprised of five vertebrae and four lumbar intervertebral discs prepared according to the previous description. The specimens were placed in the testing device, their position being conditioned by the estimated dimensional values. Thus, to calculate the load and axial resistance, the samples were placed vertically, central between the test machine ferries.

The testing was carried out by applying variable forces and supervision. The displacement interval was represented by the segment $0-10 \mathrm{~mm}$ with maintenance every $2 \mathrm{~mm}$. For each displacement interval, the force values were recorded, at the initial moment and the end of displacement, and the 'return' resistances encountered after 2, 4, 6 and $8 \mathrm{~min}$, with the registration of forces at those moments. The force values (expressed in $\mathrm{Nm}$ ) reflected the behavior of the assembly vertebrae-discs-ligaments. To differentially quantify the resistances from the column of bodies and those induced in the system by the arches column, separate testing occurred at compression, control samples, represented by ligamentous-disc-vertebral blocks (2 lumbar vertebrae and the corresponding disc) from which all the posterior arch elements were removed, mainly the zygapophyseal complex (Fig. 1).

The lateral positioning (eccentric placement) (Fig. 2) of preparation allowed the eccentric force application; thus, the flexion/extension movement could be simulated. The metric displacement monitoring followed the consistent application of a $5 \mathrm{Nm}$ equal force, quantified displacement in turn in angular values, which was expressed in degrees.

\section{Results and Discussion}

Axial loading study revealed the existence of three types of behavior concerning the lumbar segment at compression, namely:

Type I behavior at the axial loading represented the most uniform behavior type. As observed from the recorded data, the lumbar vertebral block showed a fast and pronounced adaptation after $2 \mathrm{~min}$, an increase of $30-70 \%$ of the initial values, decreasing gradually up to final values.

By the evolution model of system forces, this type of behavior is deemed optimal for intense and sudden moments and loads. At the same time, however, the decreased intensity of response in 4-8 min suggests the efficiency of such a system on time intervals of relatively low duration.

Type II was characterized by an approximately constant interval of resistances within 2-4 min. This type of behavior suggests the existence of an interval in which the resistance at axial loads remains relatively constant for a more extended period, a period in which the changes in the intervertebral disc are minimal. Type II represents the type of system able to sustain the intense loads (with absolute superior values to those from type I) for more extended periods without suffering notable distortions.

Type III represented the type of 'intermediary' or 'compromise' behavior, in which the interval of uniform resistances is the most extensive. Thus, after a substantial reduction between 0 and $2 \mathrm{~min}$, a relatively uniform interval appears between 2 and 6 min, after which the disc resistance actually 'collapses' or is markedly reduced up to the end (Fig. 3).

Type III presents the lowest values of resistance in the final stages (min 8) for all displacement intervals, except for the intervals of 0-2 min. Such behavior suggests the possibility of developing some effective resistances at the axial external loads on longer intervals. However, when these resistances are exceeded, the disc-vertebra assembly can no longer face the loads.

Regarding the distribution of the three types within the tested batch, the overwhelming percentage was represented by type III, i.e., by the most adjustable type to loads, with $55 \%$ of the cases. It was followed, in order, by type I (27\%) and type II $(18 \%)$.

Table I and Fig. 4 summarize the type of behavior of the three types suggested in terms of the forces [Newton-meters (Nm)] related to the displacement $(\mathrm{mm})$, a linear model behavior similar to those provided by literature (4).

Thus, type I constantly presents the lowest resistances, displacement of $10 \mathrm{~mm}$, and type II involves the most potent displacement forces (i.e., resistances). By contrast, type III reconfirms the position of the intermediate kind with the uniform behavior. An exciting aspect of the study was represented by the comparative analysis of how the three lumbar vertebral types behaved in the five displacement moments: $2,4,6,8$, and subsequently $10 \mathrm{~mm}$. Thus, at a displacement of $2 \mathrm{~mm}$, type I presents the lowest values and, at the same time, it 'concedes' resistance at the fastest rate, the type II resists to the highest loads, but on a relatively short interval, and type III 


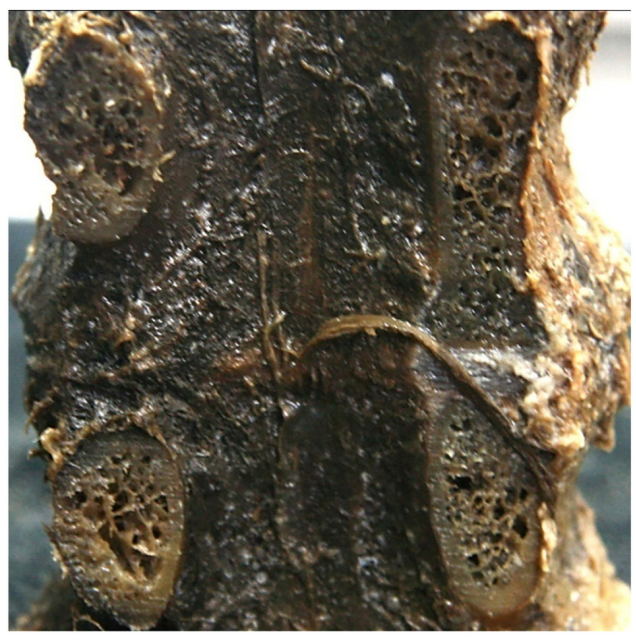

Figure 1. Vertebra with posterior arch elements removed.

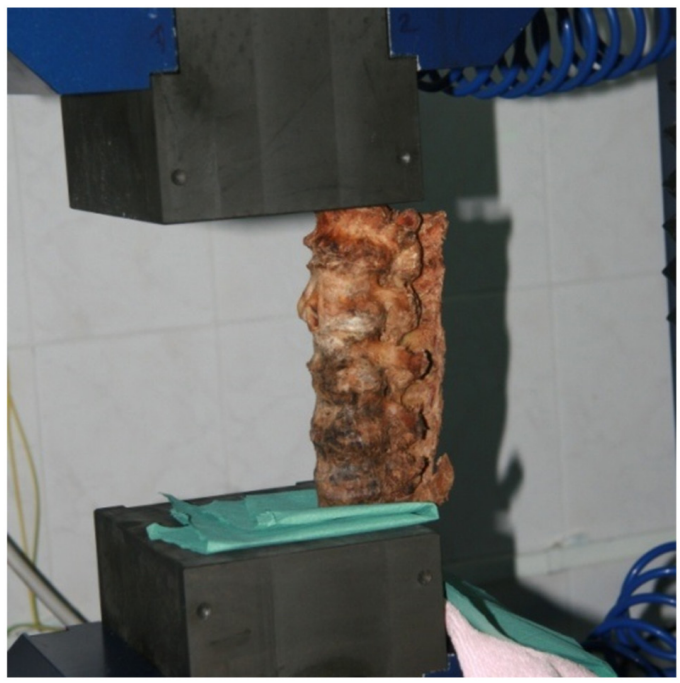

Figure 2. Lateral positioning of the vertebral segment.

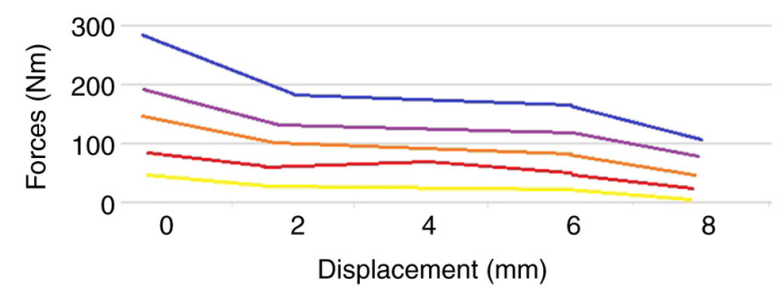

Figure 3. Axial loading type III behavior. Yellow, 2 min; red, 4 min; orange, $6 \mathrm{~min}$; purple, $8 \mathrm{~min}$; blue, $10 \mathrm{~min}$.

is constantly placed in an intermediate position, both in terms of values and in terms of dynamics. In addition, in the case of the $2 \mathrm{~mm}$ displacement, the highest value differences were recorded between the three suggested types (Fig. 5).

In the case of the $4 \mathrm{~mm}$ displacement, type I maintains the behavior model, descending steeply after min 2; type II loses the interval between min 2 and 4 but, at the same time, is reduced less while type III not only constantly maintains the trend but also manifests a slight peak at min 4. At the same
Table I. Three types of axial loading behavior.

\begin{tabular}{lccccc}
\hline Displacement $(\mathrm{mm})$ & 2 & 4 & 6 & 8 & 10 \\
\hline Type 1 & 52 & 82 & 122 & 177 & 257 \\
Type 2 & 68 & 91 & 144 & 211 & 246 \\
Type 3 & 61 & 88 & 136 & 184 & 255 \\
\hline
\end{tabular}

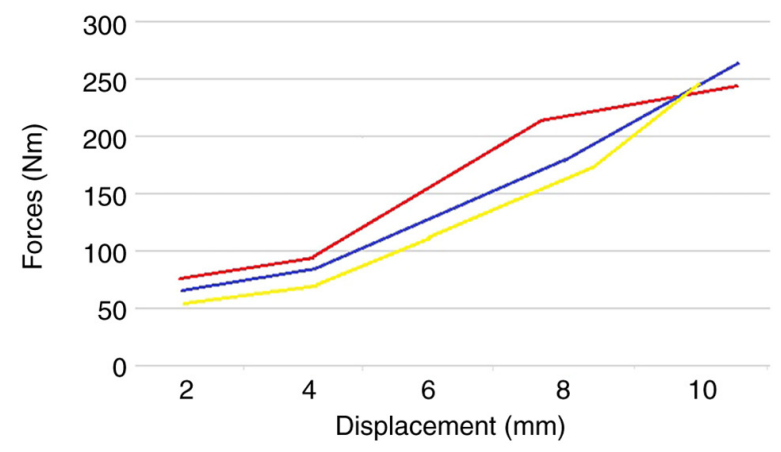

Figure 4. Forces/displacement. Yellow, type I; red, type II.

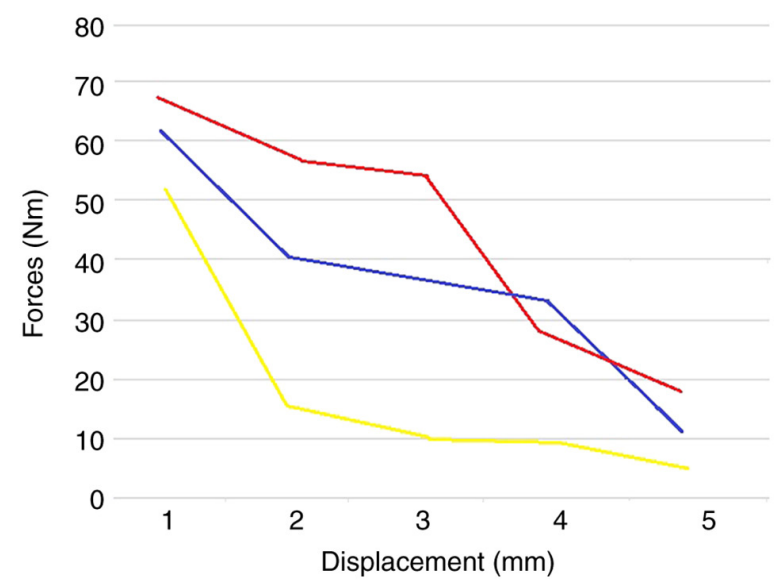

Figure 5. The 2-mm displacement. Yellow, type I; red, type II, and blue, type III.

time, the resistance of this type is decreased significantly after $\min 6$, so that, at the end of the experiment, it presents the lowest responsiveness (Fig. 6).

After compression of $6 \mathrm{~mm}$, the behavior of the three models was maintained, with two exceptions: The value difference between them was significantly low, and the interval peak disappeared from min 4 of type III. As in the previous case, at the end of the load, the resistance of type III was found to be the lowest (Fig. 7).

As the displacement interval increased $(8$ and $10 \mathrm{~mm})$, the value differences of resistances between the three types was reduced while the evolving trend remained. Moreover, type III resistance decreased below the one of type I before minute eight (Fig. 8).

This phenomenon was even more apparent at the $10-\mathrm{mm}$ displacement when the resistance of type III was the lowest after only $3 \mathrm{~min}$. Thus, at higher displacements, type III loses effectiveness. In reality, such a displacement is specifically 


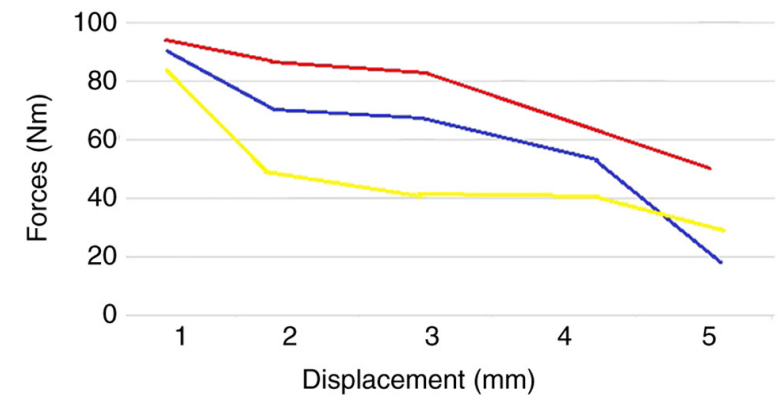

Figure 6. The 4-mm displacement. Yellow, type I; red, type II, and blue, type III.

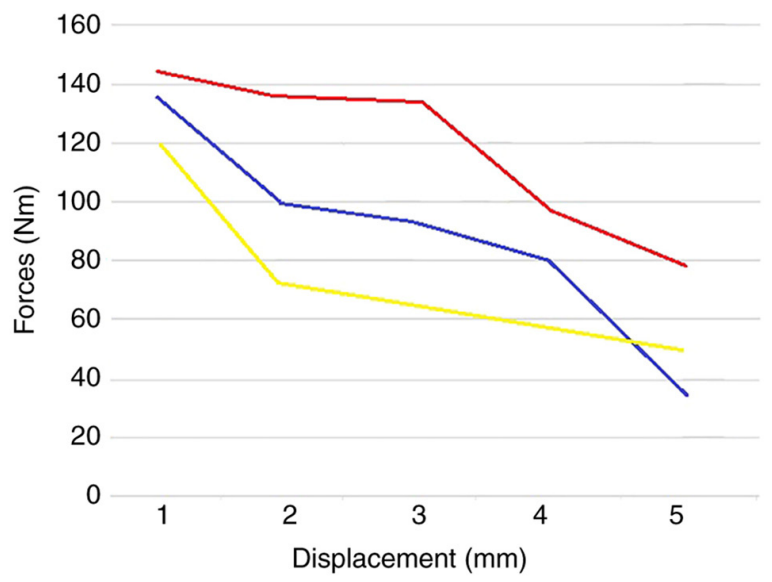

Figure 7. The 6-mm displacement. Yellow, type I; red, type II, and blue, type III.

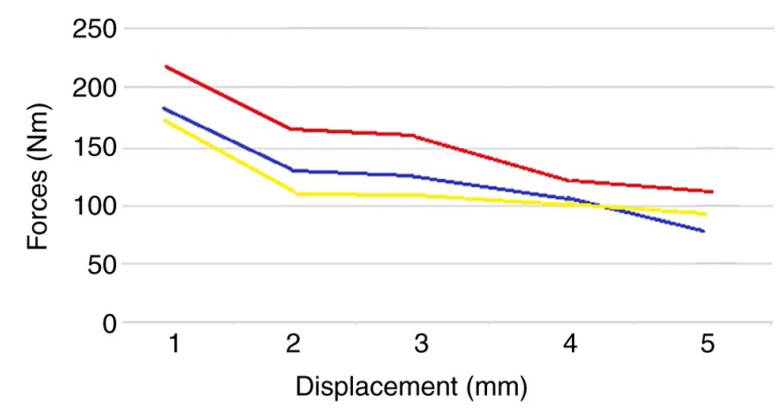

Figure 8. The 8-mm displacement. Yellow, type I; red, type II, and blue, type III.

only examined in vitro, while in vivo, such amplitudes are impossible in the case of a simple axial load, more evident in complex applications, composed and, very often, exceed physiological limits (Fig. 9).

All the above considerations support that: type I is the type of adequate response to prolonged and average to low-intensity loads; type II, responds to maximum loads, but for relatively reduced intervals; while type III is indeed the type with the uniform behavior, at least in the load margin and physiological displacements.

Eccentric loading was focused on the reproduction of the characteristic movements of flexion in the sagittal and frontal plane. The eccentric placing of the specimens allowed the bodies' axial loading to reach the edge of the column, followed by the corresponding 'pinching' of the discs in the relevant plane and correlation distances with angular values.

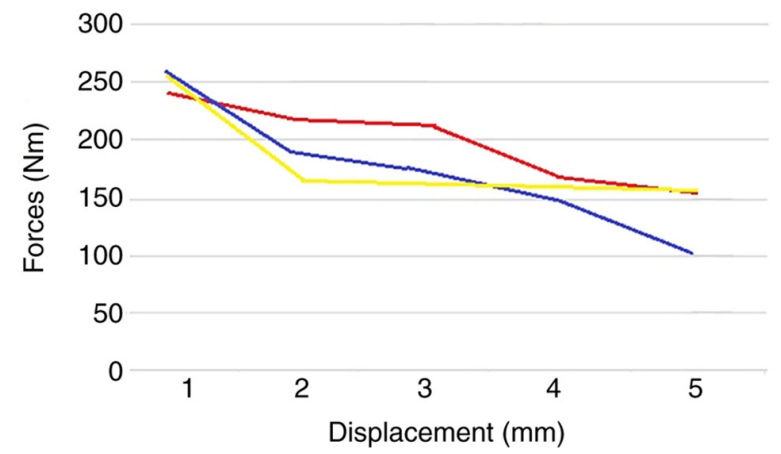

Figure 9. The 10-mm displacement. Yellow, type I; red, type II, and blue, type III.

The values obtained (Table II) are comparable to those offered by the existing literature (5), relative to the loads imposed.

It is noteworthy that the mobility in the sagittal plane (flexion earlier in our case) is much higher than that in the frontal plane, clearly indicating limited mobility by the intervertebral disc and articular complex by the presence of arches. Statistical analysis demonstrated the lack of any correlation values between the two types of movements $\left(\mathrm{R}^{2}=0.005507\right)$, underlining the absence of any elements of prediction. An interesting aspect is that correlations appeared low, statistically insignificant, even within the same movement in the sagittal plane between the two levels L1-L3 and L3-L5 $\left(\mathrm{R}^{2}=0.610427\right)$, suggesting the possibility of the emergence of significant differences in mobility between respective levels. The only values perfectly correlated were evident in the case of lateral flexion between the two analysis levels $\left(\mathrm{R}^{2}=0.938386\right)$, which suggests an element of uniformity in this type of mobility. It is an aspect worthy of considering in the analysis of phase III discopathy etiopathogeny clinically.

The calculation of loads and resistances in the vertebral-disc system has always been a concern for many authors, who employed various calculation and testing methods (6). As absolute values, in an orthostatic position, a weight of $47 \mathrm{~kg}$ presses on L4-L5 disc; with any change in the position due to muscle tension, this weight is converted into a load intervertebral disc pulpous nucleus between 282 and $726 \mathrm{~kg}$. The longer the levers and the heavier the weight to lift, the higher the loads on the pulpous nucleus, which may reach up to $1,200 \mathrm{~kg}$. In order to increase the accuracy of the determinations, center probes were placed at the level of the intervertebral discs to record intra-disc pressure. Experiments have shown that, on the third lumbar disc, on a $70 \mathrm{~kg}$ individual, each disc carries, in dorsal decubitus position, a pressure of $21 \mathrm{~kg}$, in lateral decubitus carries $70 \mathrm{~kg}$, sitting position carries $100 \mathrm{~kg}$, with trunk reclined forward at $45^{\circ}$ carries $150 \mathrm{~kg}$, and in sitting position with the trunk reclined forward at $45^{\circ}$ holding a weight of $20 \mathrm{~kg}$ pressure of $210 \mathrm{~kg} \mathrm{(6).}$

Despite the limitations, the current study on biomechanical resistance and the outcome from investigating the axial lumbar vertebral level, has highlighted several important issues.

Linders and Nuckley were among the first to study the displacement mechanism of the nucleus pulpous substance inside the IVD and found that, in the flexion of the spine, the front of adjoining vertebral plateaus approach and their rear part is separating, and the nuclear substance moves with part 
Table II. Eccentric loading values.

\begin{tabular}{lccccc}
\hline & \multicolumn{2}{c}{ Sagittal flexion } & & \multicolumn{2}{c}{ Frontal flexion } \\
\cline { 2 - 3 } \cline { 5 - 6 } $\begin{array}{l}\text { Anatomical } \\
\text { sample }\end{array}$ & L1-L3 & L3-L5 & & L1-L3 & L3-L5 \\
\hline 1 & 32.9 & 20.7 & & 29.7 & 16.4 \\
2 & 43.2 & 24.1 & & 34.5 & 18.4 \\
3 & 42.1 & 21.4 & & 33.4 & 18.6 \\
4 & 33.2 & 20.1 & & 24.6 & 13.6 \\
5 & 24.9 & 18.4 & & 35.4 & 19.7 \\
6 & 41.2 & 29.1 & & 21.5 & 11.2 \\
7 & 36.5 & 24.1 & & 28.7 & 15.4 \\
8 & 29.7 & 19.7 & & 28.4 & 13.7 \\
9 & 39.7 & 20.8 & & 24.3 & 9.1 \\
10 & 43.2 & 22.7 & & 33.5 & 20.4 \\
11 & 40.1 & 20.8 & & 30.1 & 15.7 \\
Median & 36.97273 & 21.99091 & 29.46364 & 15.65455 \\
\cline { 6 - 7 } & & & & & \\
\hline
\end{tabular}

of the mass (most) towards the rear of the IVD, albeit some of it moves towards the front, while on the right side flexion, most of the nucleus mass moves to the left and a small part of it moves to the right (7). The aforementioned studies are opposed to the hypothesis of the pulpous nucleus as a single mass, moving like a 'ball' inside the IVD.

According to this study, while the vertebral bodies are rigid systems, the pronounced reduction in the resistance system is a phenomenon that can be placed exclusively on adaptive changes that occur in the intervertebral disc. Resistance-pronounced decrease in the system within minutes is nothing but a volumetric adaptation to mechanical axial loads of the intervertebral disc. Decreased mobility in the system increases stress on the structures, while disc elasticity prevents this phenomenon. In addition, such considerations are applicable in the case of the disc at which the degenerative processes occurred $(4,8,9)$. At present, it is certain that the resistance of the disc varies depending on the degree of degeneration, leading, in turn, to a decreased resistance in cases of moderate degeneration and increased rigidity, and subsequently to a decrease of the force absorption power, in case of severe degeneration $(6,7,10-19)$. Furthermore, the parallelism between the load on the one hand, and the disc degeneration, on the other hand, can engage the fibrous annulus in a vicious cycle, which invariably results in irreversible ruptures (7).

Another aspect worth mentioning is that, while the preservation of preparations tried, as much as possible, to limit the biochemical changes that occur in vitro, the experiment was conducted at ambient temperature and not the average body temperature, which, in turn, may alter the values obtained.

In the current study, type III, the type considered most adaptable, represented the majority (55\%), i.e., more than double each of the other two types. It was followed by type I, the one that, after a swift adjustment to the initial moment, 'conceded' resistance in a uniform manner.

In conclusion, the behavior type of the monitored specimens and especially the results obtained allowed the mapping of objective parallelism between values found in the current study and behavior in vivo of the lumbar vertebral segment. A critical aspect revealing the degree of objectivity of the study is that this type of behavior manifested throughout the test interval of $10 \mathrm{~mm}$, an element confirming that preparations behaved uniformly, a biomechanical behavior induced obviously by the morphological substrate. Additionally, monitoring the behavior of the lumbar complex for long intervals of time after application of forces is an original element that allows the evaluator to determine as objectively as possible, concerning the evolution of adaptation, the likely degree of disc degeneration. Examination of disc loads and especially the behavior of vertebral segments under various load types and values can contribute on the one hand to identifying the potential etiologies of pathological manifestations at this level and, on the other hand, to the development of effective prevention and/or treatment strategies in the matter. Thus, correlating the disc behavior and the load makes biomechanics, besides a prognostic factor, a genuine therapeutic factor in disc regeneration.

\section{Acknowledgements}

Not applicable.

\section{Funding}

No funding was received.

\section{Availability of data and materials}

Not applicable.

\section{Authors' contributions}

DMI, CI, IB, PB, EG and MGI conceived and designed the study. DMI, PB, FV, SIM, MGI and BO acquired the data. DMI, PB, IB and MGI assessed the authenticity of the data. CI, IB, EG, FV and SIM analyzed the data. DMI, PB, IB, MGI and $\mathrm{BO}$ validated the results. EG, MGI, DMI, PB, FV, CI and IB were responsible for the preparation of the original draft. SIM, EG, FV and BO, were responsible for the final manuscript editing. DMI, PB, CI, IB, SIM and MGI supervised the manuscript publication. All authors read and approved the final manuscript.

\section{Ethics approval and consent to participate}

Not applicable.

\section{Patient consent for publication}

Not applicable.

\section{Competing interests}

The authors declare that they have no competing interests.

\section{References}

1. Iliescu DM, Bordei P, Ionescu EV, Albina S, Oprea C, Obada B, Lupu AA, Hangan TL and Iliescu MG: Anatomic-imaging correlations of lumbar disk-vertebral morphometric indices. Int J Morphol 35: 1553-1559, 2017. 
2. Ardeleanu V, Toma A, Pafili K, Papanas N, Motofei I, Diaconu CC, Rizzo M and Stoian AP: Current pharmacological treatment of painful diabetic neuropathy: A narrative review. Medicina (Kaunas) 56: 25, 2020.

3. Stefanescu DC, Ciucu AA, Rabinca AA, Buleandra M, Stoian AP, Jecan CR and Razvan H: An integrative medical perspective on novel dopamine detection method. Revista de Chimie 69: 277-281, 2018.

4. Kurowski P and Kubo A: The relationship of degeneration of the intervertebral disc to mechanical loading conditions on lumbar vertebrae. Spine (Phila Pa 1976) 11: 726-731, 1986.

5. Barthes X, Walter B, Zeller R and Dubousset JF: Biomechanical behaviour in vitro of the spine and lumbosacral junction. Surg Radiol Anat 21: 377-381, 1999.

6. Nachemson AL, Schultz AB and Berkson MH: Mechanical properties of human lumbar spine motion segments. Influence of age, sex, disc level, and degeneration. Spine (Phila Pa 1976) 4: $1-8,1979$.

7. Linders DR and Nuckley DJ: Deduction of spinal loading from vertebral body surface strain measurements. Exp Mechanics 47: 303-310, 2007.

8. Niosi CA and Oxland TR: Degenerative mechanics of the lumbar spine. Spine J 4 (6 Suppl): 202S-208S, 2004.

9. Hutton WC, Ganey TM, Elmer WA, Kozlowska E, Ugbo JL, Doh ES and Whitesides TE Jr: Does long-term compressive loading on the intervertebral disc cause degeneration? Spine (Phila Pa 1976) 25: 2993-3004, 2000.

10. Stoian PA, Hainarosi R, Pietrosanu C, Rusescu A, Andronache LF, Paunica S, Balalau C and Pituru TS: Modern concepts in non-surgical esthetics; a review. J Mind Med Sci 6: 190-195, 2019.

11. Best BA, Guilak F, Setton LA, Zhu W, Saed-Nejad F, Ratcliffe A, Weidenbaum M and Mow VC: Compressive mechanical properties of the human anulus fibrosus and their relationship to biochemical composition. Spine (Phila Pa 1976) 19: 212-221, 1994.

12. Gay RE, Ilharreborde B, Zhao K, Zhao C and An KN: Sagittal plane motion in the human lumbar spine: Comparison of the in vitro quasistatic neutral zone and dynamic motion parameters. Clin Biomech (Bristol, Avon) 21: 914-919, 2006.
13. Hansson TH, Keller TS and Spengler DM: Mechanical behavior of the human lumbar spine. II. Fatigue strength during dynamic compressive loading. J Orthop Res 5: 479-487, 1987.

14. Berkson M, Nachemson A and Schultz A: Mechanical properties of human lumbar spine motion segments. II. Response in compression and shear, influence of gross morphology. J Biomech Eng 101: 53-55, 1979.

15. Tofolean DE, Mazilu L, Staniceanu F, Mocanu L, Suceveanu AI, Baz R, Parepa RI, Suceveanu AP, Bondari S, Bondari D and Voinea F: Clinical presentation of a patient with Cutis Laxa with systemic involvement: A case report. Rom J Morphol Embryol 56: 1205-1210, 2015.

16. Iliescu M, Bordei P, Iliescu DM, Ciobotaru C, Lucescu V, Covaleov A and Ionescu C: Anatomo-clinical and imagistic correlations within the lumbar discopathy. Surg Radiol Anatomy 31: 145, 2009.

17. Tuta LA, Iorga I, Azis O and Voinea F: End-of-life care in patients with end-stage renal disease-ethical and clinical issues. Bk2: Political Sciences, Law, Finance, Economics and Tourism Conference Proceedings. pp487-493, 2015.

18. lliescu M, Bordei P, Albina S and Ionescu C: Morphology of the intervertebral foramen: A direct relation with low back pain. ARS Medica Tomitana 18: 62-65, 2012.

19. Moraru D, Suceveanu AP, Stoian AP, Nitipir C, Pituru S, Voinea F, Timofte D and Suceveanu AI: Amyloidosis-the importance of an early diagnosis. In: Proceeding of 35 Balkan Medical Week, 25th-27th September 2018, Athens, pp117-121, 2018.

(i) $($ This work is licensed under a Creative Commons Attribution-NonCommercial-NoDerivatives 4.0 International (CC BY-NC-ND 4.0) License. 